\title{
Research on Slow Moving Spare Parts Demand Prediction in Navigation Mark Based on SES Method
}

\author{
Feng Lei \\ Wuhan Huaxia University of Technology \\ fl_yutian@126.com
}

\begin{abstract}
This paper aims to study the slow moving spare parts demand prediction in navigation mark. Firstly, it analyzes characteristics of navigation mark spare parts and focuses on the demand prediction of slow moving spare parts. Then, SES method is adopted to study on batteries demand prediction of navigation marks in Wuhan Waterway Bureau. At last, the research method and calculating results are proved to be effective through actual application.
\end{abstract}

Keyword: Navigation mark, Slow moving spare parts, Battery, SES

\section{Introduction}

Navigation mark as quite important navigational aids wildly used in inland river and offshore bank. Meanwhile, a few of navigation marks are yearly maintained by waterway maintenance management department and result a supply problem of spare parts. Those spare parts should be divided into the fast moving and slow moving spare parts to keep navigation marks on normal working condition. Therefore, an obvious problem is always placed in front of researchers that keep lower inventory and more precise forecasting to satisfy routine work demand. Particularly, the characteristics of low frequency demand, long interval period, demand uncertainty in slow moving spare parts that usually take up a lot of inventory cost as key components ${ }^{[1]}$.

\section{Literature review}

As mentioned above, a lot of researchers have concentrated on the research field of slow moving spare parts, meanwhile, different researchers provide different names for slow moving spare parts that include intermittent demand spare part, uncertain spare parts, lumpy spare part, Irregular demand spare part and uncommonly used spare part. However, those names were given by researchers are similar to the definition of slow moving spare parts and respectively describes one characteristic of slow moving spare parts ${ }^{[2-3]}$. At the same time, for actual research condition demand that researcher should face the problems of less historical data and a larger number of value zero in the effective samples. In this research field, lots of researchers provide some different predict method that mainly include the methods of Bayesian $^{[4]}$, Croston $^{[5]}$, Bootstrap ${ }^{[6]}$, SVM and so on. Nevertheless, those research methods cannot better solve the historical data and samples prediction problem that involve a large amount of value zero. 


\section{Slow moving spare parts demand prediction}

As a commonly used method in the production prediction of exponential smoothing method, the result of test value is a weighted average of historical data, meanwhile, its weight conforms to recent large numerical weight value and forward small numerical weight value. According to previous prediction methods and research results, this paper prepares to select the method of single exponential smoothing(SES) to study slow moving spare parts demand prediction.

In accordance with the basic formula of SES, this paper assumes the time series is $y_{1}, y_{2} \ldots y_{t} \ldots$, and SES model display as follow:

$$
\lambda_{t}=a y_{t}+(1-a) \lambda_{t-1}
$$

$\lambda_{t}$ - t phase of period single exponential smoothing value;

$a$-weighting coefficient;

Based on this basic formula, an obviously expansion equation can be obtained by this study express as follow:

$$
\begin{aligned}
\lambda_{t}= & a y_{t}+a(1-a) y_{t-1}+(1-a)^{2} y_{t-2}+a(1-a)^{3} y_{t-3}+ \\
& a(1-a)^{4} y_{t-4}+\ldots \ldots+a(1-a)^{t-1} y_{1}+(1-a)^{t} \lambda_{0} \\
= & a \sum_{j=0}^{t-1}(1-a)^{j} y_{t-j}+(1-a)^{t} \lambda_{0}
\end{aligned}
$$

Because of $0<a<1$, and $t \rightarrow \infty$, a distinct result can be obtained is $(1-a)^{t} \rightarrow 0$, therefore, the formula (3) changes to formula (5).

$$
\lambda_{t}=a \sum_{j=0}^{t-1}(1-a)^{j} y_{t-j}
$$

In the following, this paper assumes the previous exponential smoothing value of $\lambda_{t}$ as the next installment value of $y_{t+1}$ 's predicted value is $\hat{y}_{t+1}$, therefore, the two equations display as follow:

$$
\begin{aligned}
\lambda_{t}= & \hat{y_{t+1}}, \lambda_{t-1}=\hat{y}_{t} \\
\hat{y}_{t+1}= & a y_{t}+a(1-a) y_{t-1}+(1-a)^{2} y_{t-2}+a(1-a)^{3} y_{t-3} \\
& +a(1-a)^{4} y_{t-4}+\ldots \ldots+a(1-a)^{t-1} y_{1} \\
= & a \sum_{j=0}^{t-1}(1-a)^{j} y_{t-j}
\end{aligned}
$$


According to this assumption, those weighting efficient of $a, a(1-a), a(1-a)^{2} \ldots \ldots$ completely obey geometric series decay. Meanwhile, this research draws following conclusions.

Because of $0<a<1$, and $a>a(1-a)>a(1-a)^{2}>\ldots \ldots a(1-a)^{t-1}$. Consequently, the sum of all weight coefficient value is 1 .

$$
\begin{aligned}
& a \sum_{j=0}^{t-1}(1-a)^{j}=1 \\
& \hat{y_{t+1}}=a y_{t}+(1-a) \hat{y_{t}}
\end{aligned}
$$

On the basis of formula (7), the slow moving spare parts SES method has been simplified. $\hat{y}_{t+1}$ and $\hat{y}_{t}$ respectively represent predicted value of period $t+1$ and actual value of period t.

\section{Example verification}

Navigation marks as the most important navigation AID wildly used in Changjiang river. This paper selects the periods of 2014 to 2016's historical consumption data of navigation mark batteries of Wuhan Waterway Bureau as research samples, the SES method studies in slow moving spare parts as displayed above is adopted by calculating application (See Table1).

Table 1 2014-2016 actual consumption of batteries

\begin{tabular}{ccccccccccccc}
\hline Month & 1 & 2 & 3 & 4 & 5 & 6 & 7 & 8 & 9 & 10 & 11 & 12 \\
Consumption & 0 & 0 & 0 & 1 & 0 & 0 & 1 & 0 & 2 & 1 & 0 & 1 \\
\hline Month & 13 & 14 & 15 & 16 & 17 & 18 & 19 & 20 & 21 & 22 & 23 & 24 \\
Consumption & 0 & 0 & 1 & 0 & 0 & 1 & 0 & 0 & 2 & 0 & 1 & 0 \\
\hline Month & 25 & 26 & 27 & 28 & 29 & 30 & 31 & 32 & 33 & 34 & 35 & 36 \\
Consumption & 0 & 0 & 0 & 1 & 0 & 0 & 1 & 0 & 0 & 1 & 0 & 0 \\
\hline
\end{tabular}

In the following calculation, this paper assigns values of $\alpha$ and $\hat{y}_{\mathrm{t}}$ respectively. Then, its assume the values of $\alpha=0.1,0.2,0.3,0.4,0.6,0.9$ and $\hat{y}_{t}=0,1,2$ in SES forecasting process. From those analysis diagrams display as follow, the relationship among weighted value, original value with sample have obtained by researcher. According to figure 1 (a),(b),(c) and (d), the outcomes calculated by weighted values have a larger effect on the first 20 group classification historical consumption data. At the rest of data from period 21 to 36, the predictive value curve increasingly overlap at one point if its have enough group classification samples. With increasing of weighed value, both weighted value and original value are produced less influence on outcomes in the diagram Fig. 1 (e) and (f) especially. Therefore, the different original values in a larger number of samples result a relative low influence to final predictive values in a reasonable weighted value. 


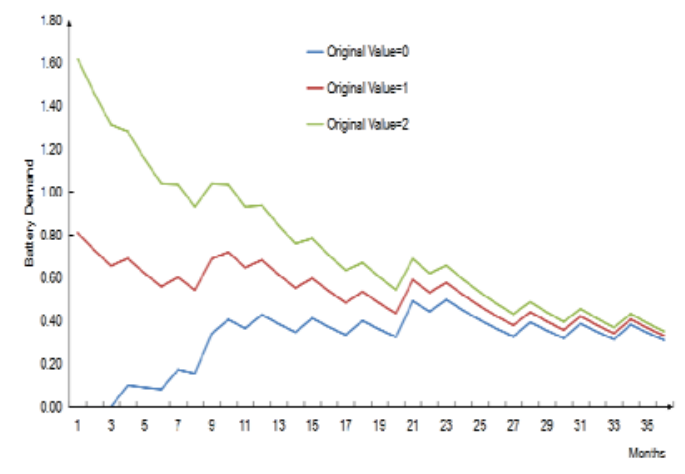

weighted value $0.1(\mathrm{a})$

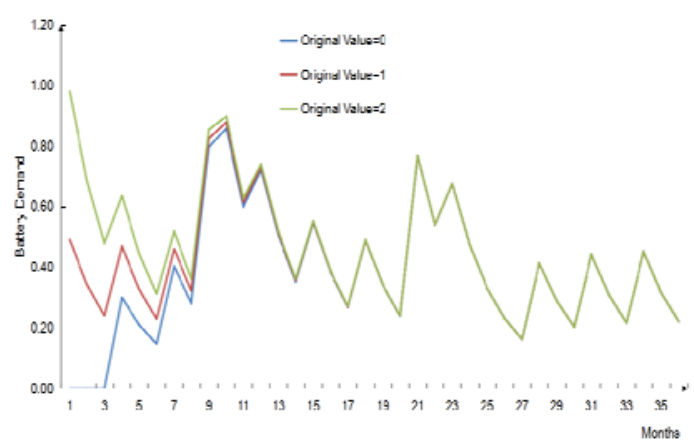

weighted value $0.3(\mathrm{c})$

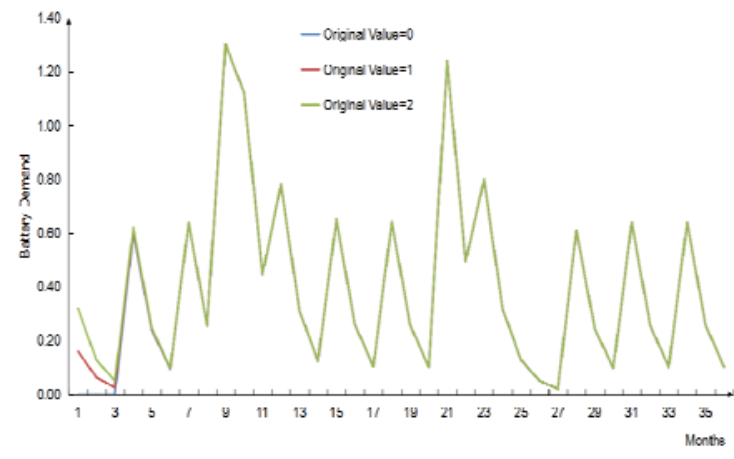

weighted value $0.6(\mathrm{e})$

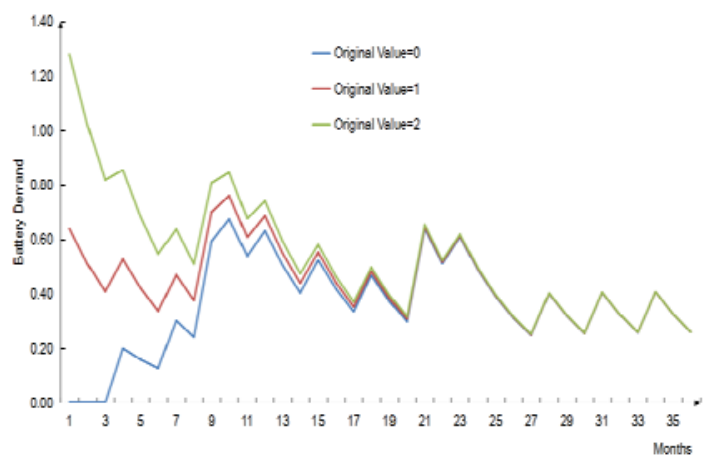

weighted value $0.2(\mathrm{~b})$

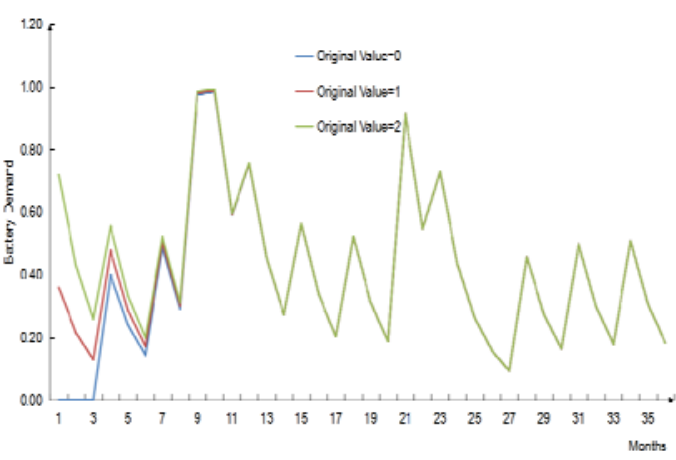

weighted value $0.4(\mathrm{~d})$

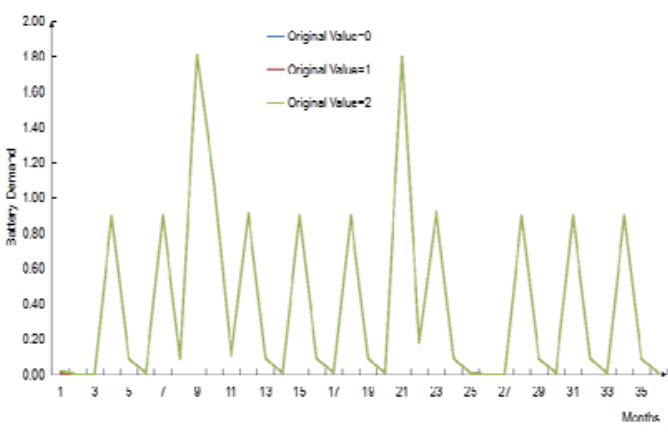

weighted value $0.9(\mathrm{f})$

Figure 1 The Original value analysis diagram in different weighted values

As this paper mentioned above, the original value rarely produces influence on predicting results and weighted value $\alpha$ should be confirmed by following study. Based on those calculate results and diagrams, the study selects the method of variance calculation by predicted value and actual value to analyze and confirm a reasonable weighed value. In terms of variance outcomes in table 2 , this study provide an obvious result that $\alpha=0.9$ produce a minimum variance in those weighted values. Thereby, 0.9 is adopted by SES method as reasonable weighted value.

Table 2 Variance of predicted value and actual value

\begin{tabular}{ccccccc}
\hline Weighted & $\alpha=0.1$ & $\alpha=0.2$ & $\alpha=0.3$ & $\alpha=0.4$ & $\alpha=0.6$ & $\alpha=0.9$ \\
Variance & 0.32417 & 0.26608 & 0.21764 & 0.17285 & 0.09195 & 0.00790 \\
\hline
\end{tabular}




\section{Conclusion}

Finally, in the samples of 2014 to 2016 group classification historical data, the weighted value of 0.9 conforms to relatively stable time series samples. On the contrary, smaller weighted value can produce a relatively reasonable predicting results in volatile time series samples. Meanwhile, in order to satisfy a lot of discrete random demand, the simplex SES method can predicts spare parts consumption in larger group classification historical data, but rarely satisfy the no historical data and the multi-effects factors’ predict demand.

\section{Reference}

[1] Haneveld W K K, Teunter R H. Optimal provisioning strategies for slow moving spare parts with small lead times[J]. Journal of the Operational Research Society, 1997, 48(2):184-194.

[2] Cui N F, Ding L M. An Inventory Model of Slow Moving Spare Parts Based on Function of Parts' Life-cycle[J]. Systems Engineering, 2006.

[3] Zeng Y, Wang L. A hybrid decision support system for slow moving spare parts joint replenishment: a case study in a nuclear power plant[J]. International Journal of Computer Applications in Technology, 2010, 37(3/4):287-296.

[4] Kamath K R, Pakkal T P M. A Bayesian approach to a dynamic inventory model under an unknown demand distribution[J]. Computers \& Operations Research, 2002, 29(4):403-422.

[5] Croston J D. Forecasting and Stock Control for Intermittent Demands[J]. Journal of the Operational Research Society, 1972, 23(3):289-303.

[6] Lawrence M. Commentary on: A new approach to forecasting intermittent demand for service parts inventories[J]. International Journal of Forecasting, 2004, 20(3):389-390. 